\title{
Approaches to building rapport with patients
}

\author{
Author: Mohsin F Butt ${ }^{\mathrm{A}}$
}

In this article, I detail my rapport-building trial and error exercise and outline why addressing a patient's occupation at the beginning of a consultation worked during my history taking. Context permitting, addressing occupation can be useful when setting the tone and rhythm of a conversation, helping to focus explanations on the back of a patient's everyday experiences, and allowing a history-taker to address a patient's ideas, concerns and expectations more comfortably. Broaching occupation can also be a useful way to extrapolate information about disease risk factors and baseline understanding from the start. Whatever the rapportbuilding technique used, self-awareness seems to be a critical skill in this exercise, and an approach used with one patient may not necessarily be useful with another.

KEYWORDS: clinical competence, education, medical education, instruction, medical curriculum

DOI: $10.7861 /$ clinmed.2021-0264

As a foundation year-1 doctor working during the COVID-19 pandemic, the need to fill rota gaps while staff have been selfisolating has deprived me of forming meaningful doctor-patient relationships. Anecdotally, patients have been frustrated with the lack of continuity of care and feel isolated and confused amid the conveyer belt of junior doctors that see them. The need to efficiently build rapport with patients is pressing.

Rapport is defined as a 'harmonious relationship' and relates to collaboration and parity between patient and physician. Effective rapport has been shown to improve patient compliance with treatment, clinical outcomes and patient satisfaction. ${ }^{2}$ Mehrabian and Ferris reported that impactful communication (the cornerstone of effective rapport building) was $7 \%$ verbal and $93 \%$ non-verbal, where the non-verbal component was composed of body language $(55 \%)$ and tone of voice $(38 \%))^{3}$ Unfortunately, their report pre-dated the wearing of personal protective equipment and social distancing in the COVID-era.

New to clinical medicine, and as someone who relies on facial expressions as my primary tool for communication, I have had to adapt the skills I was taught as an undergraduate during

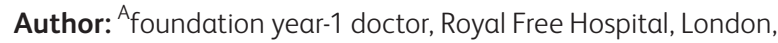
UK and Barts and The London School of Medicine and Dentistry, London, UK sessions with simulated patients. ${ }^{4}$ Wearing a mask that covered half my face, I realised that establishing rapport through verbal communication was of increasing importance. I trialled three approaches. First, I elicited a patient's ideas, concerns and expectations at the beginning of a consultation but I soon realised I had limited time, particularly on a hectic acute medical unit shift, to respond sensitively and allay a patient's fears. Without time, this approach felt like a tick-box exercise and I was faced with the 'well, you're the doctor' statement far too many times for my liking. Secondly, I moved towards a flat hierarchy by presenting myself to patients with my first name only, but soon realised that introducing myself with my surname, Dr Butt, was more memorable for patients, particularly on my gastroenterology rotation. Thirdly, I decided to pay more attention to a patient's social history, particularly their occupation, later experimenting with including this at the beginning of a consultation, with surprisingly positive results.

Patients seemed consistently pleased that their career (an element of their personal life that they had committed over $60 \%$ of their waking day to) had been broached. Herein, I detail my experiences of asking patients about their career status, particularly as a consultation-starter, and suggest an approach that other house officers may consider using.

Used with caution and never wholly relied upon, querying a patient's career at the beginning of a consultation has helped me to set the pace of a conversation, allowing me to extrapolate a patient's potential health awareness, baseline medical understanding and ability to process new concepts and complex explanations. Moreover, asking about occupation has helped me focus my explanations on the back of a patient's everyday experiences, allowing me to address a patient's ideas, concerns and expectations more comfortably throughout the course of the consultation. For instance, during an encounter with a retired consultant anaesthetist, I found little need to employ layman's terms to describe medical terms and the future management plan. Similarly, during my vascular surgery placement, I found negotiating an explanation of a surgical procedure to be slightly easier when a patient worked as a tradesperson compared with a professor of history, for instance. Lastly, addressing occupation from the beginning ensures occupational risk factors are not forgotten, such as exposure to second-hand smoke, asbestos and stress (in the case of anyone who works in the NHS).

The approach I have described relies on potential preconceptions that one holds about a particular person or personality that is drawn to a profession, and history-takers should be wary of their own unconscious biases that could inadvertently damage rapport. Moreover, although it is a window into someone's interests and life, 
it is important to remember that a career is not entirely reflective of one's entire being. Adopting the 'patient role' and being placed in a position of vulnerability may also change one's mindset and cloud a patient's baseline level of understanding and ability to process new, complex information. As such, clinicians should ensure they remain sensitive to verbal and non-verbal cues of a patient's uncertainty.

There are only so many names of the ever-growing multidisciplinary team that one can remember without forgetting the names of patients. Working on a vascular surgery ward, I have found it challenging to remember the name and medical history of a patient with an ischaemic leg being treated with a similar cocktail of drugs to the patient in the next bay. For me, it has been much easier to remember a patient's history when I think of them as 'the foreign affair correspondent / music teacher / tradesperson with $X$ disease'. For me, humanising a patient through the lens of their career is so much easier, although I am conscious of the limited number of careers that we can remember before being afflicted with the same memory fatigue that comes with remembering names. Although, that said, I find learning careers to be more interesting than remembering a list of story-less names or bed numbers.

Admittedly, asking about current or former career at the beginning has been met with looks of confusion by a minority of patients. I propose several hypotheses: the slightly blunt way in which I may have posed the question; a patient thinking 'What does this have to do with the medical history?'; a patient thinking 'Is this doctor actually interested in my life?'; and a patient thinking 'This is not the normal structure of a history,' for those who have been seen by several different doctors and are accustomed to the standard pro forma.

Through trial and testing, I have realised that the aforementioned issues are easily rectified by providing some context to the question, such as 'to learn a little about your life story, could you start with telling me what you do / have done for a living?'. This is loaded with genuine interest and puts the ball in the patient's court from the beginning, helping re-enforce the doctor-patient relationship. From my limited experience, this has generally been met with gratitude and injects mutual respect throughout the remainder of the conversation. Carefully phrased in such a way, and to single out one group of patients in particular, I have found those labelled with medically unexplained diagnoses (a highlight of the January 2021 issue of this journal) who may feel ignored by the medical establishment to be particularly receptive to such a questioning style. ${ }^{5}$

Admittedly, there have been instances where I have unknowingly asked about occupation to unemployed patients, but from my experience, this has never been met with hostility given that I intended no malice. I am also wary that unemployed patients, homeless patients or those with chronic debilitating conditions may begin to fear interactions with healthcare professionals if occupation is routinely probed at the beginning of every medical interaction. I have learned that context and self-awareness (a skill we should all be continuously refining) are critical to any rapport building exercise. Inevitably one may encounter a patient whose job one knows little about and for whom brief follow-up questions are difficult to conjecture. I can think of few scenarios where words to the effect of 'thank you for sharing that' cannot be employed.

Through probing a patient's career, among some of the most interesting patients I would otherwise not have truly understood include a concert pianist, a school librarian, a martial arts teacher and a poet. One of my most memorable house officer experiences to date occurred when I put aside a few minutes at the end of the day to listen to the pianist's audio rendition of Rachmaninoff's Piano Concerto No 2. Overheard by patients in adjacent beds, this lit up the patient bay and brought together a group of patients who had previously known very little about each other's lives. Music can lead people to friendship.

Context considering, career probing, particularly at the beginning of a medical history, has helped me efficiently and effectively establish rapport with my patients. Rapport building is not an exact science and part of the joy of this trial-and-error exercise was understanding the techniques that worked for me and adapting those approaches to the patient in front of me. Regardless of the technique used, selfawareness seems to be an important skill for this exercise, and an approach used with one patient may not necessarily be useful with another. I sincerely hope this piece has inspired fellow house officers to trial their own rapport building approaches.

\section{References}

1 Spink LM. Six steps to patient rapport. AD Nurse 1987;2:21-3.

2 Leach MJ. Rapport: a key to treatment success. Complement Ther Clin Pract 2005;11:262-5.

3 Mehrabian A, Ferris SR. Inference of attitudes from nonverbal communication in two channels. J Consult Psychol 1967;31:248-52.

4 Butt MF. Simulated patients uncovered. Clin Teach 2018;15:81-2.

5 Emmanuel A. Finding your own way to manage patients with functional disorders. Clin Med 2021;21:1.

Address for correspondence: Dr Mohsin F Butt, The Wingate Institute of Neurogastroenterology, Barts and The London School of Medicine and Dentistry, Queen Mary University of London, 26 Ashfield Street, Whitechapel, London, E1 2AT, UK. Email: mohsin.butt@nhs.net Twitter: @ThisIsButt 